\title{
Repercussões celulares e imunológicas dos isômeros de ringer lactato na ressuscitação volêmica humana
}

\author{
Cellular and immunological effects of ringer's lactate isomers in human volume resuscitation
}

\author{
Leonardo Monzo', Marise Maleck².
}

Como citar esse artigo. Monzo L, Maleck M. Repercussões celulares e imunológicas dos isômeros de ringer lactato na ressuscitação volêmica humana. Revista Saúde. 2015 Jan./Jun.; $06 \quad$ (1): 15-19.

\begin{abstract}
Resumo
A hemorragia é responsável por 40\% das mortes por trauma e é intenso o debate sobre o manejo correto destes pacientes. O Ringer Lactato é a solução considerada ideal para ressuscitação volêmica humana. Contudo, mesmo uma substância consagrada como o Ringer Lactato promove uma série de efeitos celulares e imunológicos deletérios ao organismo. O presente estudo propôs uma revisão literária acerca da atividade dos isômeros da solução de Ringer Lactato no organismo humano com o objetivo de entender melhor a ação destes isômeros nos mecanismos imunológicos a fim de minimizar os efeitos colaterais decorrentes de sua utilização. Tal revisão se deu através de pesquisa sistemática em bases de dados na área da saúde (Medline, Lilacs, Bireme, Pubmed e Scielo.), contemplando as publicações científicas dos últimos 50 anos. Palavras-chave: Hemorragia. Ressuscitação. Ringer lactato. Isômeros.
\end{abstract}

\begin{abstract}
The bleeding is responsible for $40 \%$ of the deaths from trauma and the debate on the correct management of these patients is intense. The Lactate Ringer solution is considered ideal for volume resuscitation. However, even a substance consecrated as the Ringer Lactate promotes a series of cellular and immunological effects harmful to the body. This study proposed a literary review on the activity of isomers of Lactate Ringer solution in the human body in order to better understand the action of these isomers in immunological mechanisms to minimize the side effects resulting from its use. Such review will be through systematic research on health databases (Medline, Lilacs, Scielo, PubMed and Bireme.), including scientific publications of the last 50 years. Keywords: Hemorrhage. Resuscitation. Ringer lactate. Isomers.
\end{abstract}

\section{Introdução}

A hemorragia corresponde a $40 \%$ das mortes decorrentes de eventos traumáticos [Sauaia et al., 1995]. E a maioria dessas perdas ocorre nas primeiras seis horas após a injúria [Shackford et al., 1993]. Analisando-se as estatísticas de origem militar, observa-se que grande parte dos óbitos é plenamente evitáveis com medidas básicas de ressuscitação volêmica [Dillon et al. 1966; Shires e Canizaro 1973].

O debate acerca da solução ideal a ser utilizada na reposição volêmica humana nos eventos hemorrágicos tem sido vigorosa desde o início da segunda metade do século XX. O intervalo entre o dano e o tratamento definitivo da fonte do sangramento é crítico e as substâncias utilizadas na ressuscitação volêmica interagem com vários elementos orgânicos da circulação. Assim, a necessidade do conhecimento sobre o comportamento desses compostos quando infundidos no organismo e suas repercussões no quadro clínico do paciente é crucial [Traverso et al. 1986; Healey et al. 1998].

O choque circulatório consiste em um evento muito mais complexo do que os sinais clínicos oriundos da hipovolemia. Existe uma relação direta entre tais sinais e os eventos químicos, celulares e imunológicos que ocorrem no organismo. Portanto, faz-se necessário um entendimento destes processos desencadeados pela hipoperfusão tecidual e de como interage neste universo os isômeros opticamente ativos da mistura racêmica de Ringer Lactato, solução consagrada na ressuscitação volêmica humana.

Este estudo teve por objetivo realizar uma revisão de literatura acerca da atividade da solução de Ringer Lactato na ressuscitação volêmica humana e os efeitos colaterais, celulares e imunológicos, da mistura racêmica desse composto, expondo as vantagens biológicas da utilização da porção levogira dessa mistura.

1. Universidade Severino Sombra, Centro de Ciências da Saúde, Medicina, Vassouras-RJ, Brasil.

2. Universidade Severino Sombra, Centro de Ciências da Saúde, Mestrado Profissional em Ciências Ambientais, Vassouras-RJ, Brasil. 


\section{Os ensinamentos da guerra}

Durante a guerra do Vietnã, muitos estudos sobre este tema foram desenvolvidos e chegou-se a conclusão de que uma estratégia de reposição volêmica utilizandose de uma solução cristalóide isotônica na proporção 3:1, realizada com grandes volumes objetivou uma oferta hidroeletrolítica abundante a fim de repor perdas intravasculares e intersticiais [Shires e Canizaro 1973].

Muitos problemas foram evidenciados durante a aplicação dessa estratégia proposta no início dos anos 70. As primeiras constatações foram o edema tecidual oriundo da infusão de grandes volumes e as injúrias pulmonares decorrentes da congestão dos microcapilares e processo inflamatório subsequente [Ashbaugh et al. 1967, Demling 1980], processo este clinicamente conhecido como Síndrome da Angústia Respiratória do Adulto (SARA).

No período pós-guerra do Vietnã, nas décadas de 80 e 90, estudos realizados in vivo evidenciaram uma alta incidência de acidose metabólica hiperclorêmica e aumento na mortalidade em cobaias reanimadas com grandes volumes de solução isotônica de cloreto de sódio. Tal fato alavancou, nesse período, a solução de Ringer Lactato à condição de cristalóide de escolha na ressuscitação volêmica [Traverso et al. 1986, Healey et al. 1998].

Em 1997, a abordagem ao paciente com sinais de choque hemorrágico foi padronizada pelo Comitê do Trauma do Colégio Americano de Cirurgiões em seu curso denominado Advanced Life Trauma Support $(A T L S)$, preconizando infusão rápida de $2 \mathrm{~L}$ de Ringer Lactato em pacientes que apresentassem instabilidade hemodinâmica severa [ATLS 1997].

Nas décadas subsequentes, com o franco avanço tecnológico, estudos mais complexos foram realizados e novos efeitos sistêmicos originados da reposição volêmica com grandes volumes de cristalóides foram constatados, incluindo aumento do volume intersticial nos tecidos viscerais e cardíacos [Moon et al. 1994], síndrome compartimental abdominal [Maxwell et al. 1999 e Madigan et al. 2008], síndrome compartimental em extremidades sem injúrias [Ablove et al. 2006] e derrame pericárdico [Hashim et al. 2002]. Uma revisão mais recente ressalta, além dos efeitos clínicos deletérios, os danos celulares, metabólicos e imunológicos originados da ressuscitação volêmica agressiva [Cotton et al. 2006].

Com os crescentes relatos de complicações oriundas da reposição volêmica preconizada, atentou-se para a possibilidade de realizar tal procedimento com volumes reduzidos. As soluções salinas hipertônicas, concentradas a 7,5\%, se mostraram muito promissoras para substituir os cristalóides isotônicos, pois apresentavam maior rapidez na expansão do volume plasmático e com volumes consideravelmente menores tanto em relação à solução salina quanto ao Ringer Lactato [Velasco et al. 1980, Stanford et al. 1989].

Foi proposta também uma solução que apresentasse, concomitantemente, propriedades hipertônicas e hiperoncóticas: a solução salina hipertônica a 7,5\% adicionada de dextrana a 6\% [Velasco et al. 1989 e Chudnofsky et al.1989]. Contudo, esta solução, a princípio ideal para o atendimento préhospitalar, mostrou-se decepcionante, pois estudos norte-americanos do National Lung, Heart and Blood Institute evidenciaram um aumento na mortalidade precoce e não se mostrou capaz de superar as taxas de mortalidade obtidas com o tratamento convencional com cristalóides isotônicos [NHLBI 2009].

\section{O conhecimento atual do choque circulatório}

O decréscimo do volume da macrocirculação decorrente da hemorragia se manifesta através da hipotensão sistêmica e hipoperfusão periférica [Nunn e Freeman 1964].

A hipoperfusão na macrocirculação transmitese à microcirculação, que se traduzem uma condição de hipóxia, situação que acarreta um desarranjo na membrana mitocondrial, ativando receptores gama ativadores de peroxissomos e NADPH oxidase, enzima responsável por catalisar a transferência de um elétron do NADPH para o oxigênio molecular, formando um ânion superóxido $\left(\mathrm{O}_{2}^{-}\right)$. Esse fenômeno é responsável por intensa injúria oxidativa aos tecidos [Abdelrahman et al. 2004, Abdelrahman et al. 2005].

O distúrbio circulatório causado pelo choque hemorrágico é acompanhado de um desequilíbrio imunológico. O evento traumático é o gatilho de uma série de reações do sistema imune que aumentam a resposta inflamatória mediada por segundos mensageiros [McCloskey et al. 2004, Sato et al. 2005, Sato et al. 2007], expressão gênica [Alam et al. 2002] e ativação neutrofílica [Xu et al. 2004].

\section{Efeitos celulares e imunológicos do Ringer lactato}

Dentre os cristalóides isotônicos, o Ringer Lactato é a substância mais estudada, pois seu papel nas disfunções inflamatórias e imunes do choque hemorrágico precisa ser elucidado.

Em estudos animais, o Ringer Lactato está relacionado a um aumento na expressão neutrofílica de selectinas $\mathrm{P}$ e L, moléculas de adesão intercelular 1 (icam-1) e moléculas de adesão de células vasculares 1 (vcam-1) [Alam et al. 2000]. Em vigência de seu 
uso, observa-se também um incremento dos sítios neutrofílicos de ligação para as integrinas $\mathrm{CD} 11 \mathrm{~b}$ e CD18, diretamente envolvidas nos processos de adesão leucocitária ao endotélio vascular e estímulo da injúria oxidativa em neutrófilos [Rhee et al. 1998, Alam et al. 2004].

O Ringer Lactato ainda estimula a apoptose dos enterócitos, hepatócitos e pneumócitos tipo II e causa um decréscimo de populações celulares incluindo macrófagos, células endoteliais, epiteliais e de músculo liso [Deb et al. 1999, Deb et al. 2000, Ayuste et al. 2006].

Portanto, constatou-se que o desequilíbrio imunológico e as injúrias celulares são diretamente dependentes da substância que é utilizada na reposição de volume, com especial destaque para o Ringer Lactato, responsável por aumentar a expressão de genes em vários tipos celulares do organismo [Alam et al. 2000].

\section{Aspectos moleculares da solução de ringer lactato}

A despeito das descobertas sobre os danos causados pelo uso da solução de Ringer Lactato, esta substância ainda é preconizada pelo ATLS desde os anos 80 até os dias atuais, como primeira escolha para a ressuscitação volêmica humana, e utilizada largamente na maioria dos serviços de atendimento de emergências clínico-cirúrgicas [ATLS 1997].

Diante disso, fez-se mandatória uma intensa pesquisa para elucidar a origem citotóxica da mistura racêmica que compunha a solução de Ringer Lactato. Concluiu-se que a porção dextrogira da solução era responsável pela toxicidade. $\mathrm{O}$ isômero $\mathrm{D}$ aumenta o estresse oxidativo dos neutrófilos motivado pela produção excessiva de espécies reativas derivadas do oxigênio molecular (superóxido) [Rhee et al. 1998], além de incrementar a síntese de proteínas próapoptóticas, interferirem no fenômeno de migração celular [Koustova et al. 2002, Jaskille et al. 2004 e Jaskille et al. 2006] e aumentar a expressão gênica de mediadores inflamatórios [Alam et al. 2000].

$\mathrm{O}$ isômero L do lactato confere proteção imune, atenuando a ativação neutrofílica, além de promover a diminuição da expressão gênica de mediadores inflamatórios e da síntese de proteínas pró-apoptóticas [Koustova et al. 2002, Jaskille et al. 2004, Jaskille et al. 2006, Ayuste et al. 2006].

\section{Alternativas à Reposição Volêmica Tradicional}

Algumas possibilidades têm sido estudadas no intuito de promover uma reposição volêmica cada vez mais livre de efeitos colaterais e protetora frente aos eventos danosos decorrentes do choque circulatório. Dentre as substâncias pesquisadas, as mais promissoras são os Carreadores de Oxigênio Baseados na Hemoglobina e os Inibidores da Desacetilase das Histonas.

Os carreadores de oxigênio fazem parte de uma estratégia de terapia por substitutos sanguíneos baseados na hemoglobina, sendo o expoente desta classe o glutaraldeido polimerizado, um produto da hemoglobina bovina. Em alguns estudos esse composto demonstrou atividade imunomodulatória, diminuindo a migração e o acúmulo de neutrófilos no tecido pulmonar, além de apresentar baixo potencial imunogênico e inflamatório [Masuno et al. 2005, Dong et al. 2006]. Em modelos animais, demonstrou atividade vasoconstritora e aumento da PAM mais efetivo que a obtida tanto com cristalóides quanto com colóides isotônicos [Boura et al. 2003, Knudson et al. 2003], porém seu uso ainda carece de estudos em humanos.

Já os inibidores da desacetilação de histonas são promissores, pois podem agir na acetilação das histonas reguladoras da transcrição dos genes envolvidos nos mecanismos de defesa celular, gravemente afetados durante o estresse causado pelo choque hemorrágico e pela ressuscitação volêmica. Mas, assim como os carreadores de oxigênio, necessitam de estudos mais consistentes, sobretudo em pacientes com quadros mais graves.

\section{Material e Métodos}

O método utilizado para a revisão foi à pesquisa sistemática às bases de dados eletrônicos mundialmente consagrados (BIREME, LILACS, PUBMED e SCIELO), no período de agosto a dezembro de 2012, contemplando publicações científicas em periódicos devidamente indexados que versam sobre o tema proposto no período compreendido entre 1960 e 2010.

\section{Discussão e Conclusão}

Como foi visto, a hemorragia é a principal causa de mortes decorrentes de traumas, tanto em ambientes civis quanto militares, e durante os últimos 50 anos a discussão sobre a forma ideal de manejo destes pacientes foi bem intensa.

A reposição volêmica agressiva com cristalóides isotônicos foi, por muito tempo, a alternativa mais viável para a ressuscitação volêmica humana. Porém, estudos mais recentes mostraram que as reposições com volumes reduzidos e guiadas por metas são mais eficientes e menos deletérias que as estratégias propostas por estudos anteriores.

Tais conclusões são possíveis graças ao entendimento de que o choque circulatório não é um 
fenômeno restrito à macro circulação e aos sinais exibidos no quadro clínico sindrômico.A hipóxia causada pela hipoperfusão tissular leva a um desequilíbrio imunológico e celular, desencadeando uma produção em massa de mediadores inflamatórios, proteínas de atuação em nível endotelial entre outras, promovendo uma série de reações danosas ao organismo.

Muitas substâncias foram testadas, na esperança de encontrar um composto que apresentasse a capacidade de promover uma reposição volêmica adequada sem efeitos colaterais relevantes. Descobriu-se que o componente levogiro da mistura racêmica de Ringer Lactato, além de não apresentar efeitos colaterais significativos relacionados ao seu uso, confere ainda proteção efetiva contra as injúrias imunológicas e celulares acarretadas pela hipoperfusão tecidual no choque hipovolêmico.

Deve-se lembrar que, em hipótese nenhuma, as estratégias de ressuscitação volêmica humana, quaisquer que sejam, constituem tratamento definitivo do choque hipovolêmico. Tais procedimentos têm seu relevante papel na manutenção de uma perfusão adequada de estruturas nobres, como o Sistema Nervoso Central, coração e rins, por exemplo. Todos os esforços devem ser concentrados na detecção e tratamento precoce do foco primário de sangramento.

Atualmente, uma reposição feita apenas com a porção levogira de Ringer Lactato parece ser a indicada para atingir as metas de baixa toxicidade e diminuição dos efeitos colaterais. Contudo, a separação da mistura racêmica ainda não se constitui em um método que torne seu uso mais viável. Além disso, a excelência da solução de Ringer Lactato e os índices de sucesso na ressuscitação volêmica realizada com esta substância fazem com que os esforços no intuito de sintetizar o isômero levogiro sejam arrefecidos.

Umaperspectiva interessante paranovas pesquisas reside nos fármacos que se mostram promissores adjuvantes na redução dos efeitos colaterais oriundos da reposição volêmica com as substâncias disponíveis, com especial atenção para os inibidores da desacetilação das histonas. Essas drogas já se encontram em fases 1 e 2 de testes mas seu uso ainda carece de estudos mais consistentes em situações de traumas mais extensos.

Por agora, uma reposição volêmica eficiente, guiada por metas e com volumes modestos, além da detecção e tratamento precoce da fonte do sangramento, ainda constitui-se nas estratégias mais eficientes a serem seguidas.

\section{Referências}

Abdelrahman M, Collin M, Thiemermann C: The peroxisome proliferatoractivated receptor-gamma ligand 15-deoxydelta12, 14-prostaglandin J2 reduces the organ injury in hemorrhagic shock. Shock 22:555-561, 2004.

Abdelrahman M, Mazzon E, Bauer M, Bauer I, Delbosc S, Cristol JP, Patel NS, Cuzzocrea S, Thiemermann C: Inhibitors of NADPH oxidase reduces the organ injury in hemorrhagic shock. Shock 23:107-114, 2005.
Ablove RH, Babikian G, Moy OJ, Stegemann PM: Elevation in compartment a pressure following hypovolemic shock and fluid resuscitation: a canine model. Orthopedics 29:443-445, 2006.

(ATLS) Advanced Trauma Life Support for Doctors. Chicago, IL: American College of Surgeons Comite on Trauma, 1997.

Alam HB, Santo K, Koustova E, Buris D, Riche N, Rhee P: Defect of different resuscitation strategies on neutrophil activation in a swine model of hemorrhagic shock. Resuscitation 60:91-99, 2004

Alam HB, Stegalkina S, Rhee P, Koustova E: cDNA array analysis of gene expression following hemorrhagic shock and resuscitation in rats. Resuscitation 54:195-206, 2002.

Alam HB, Sun L, Ruff P, Austin B, Buris D, Rhee P: E- and P-selectin expression depends on the resuscitation fluid used in hemorrhaged rats. J Surg Res 94:145-152, 2000.

Ashbaugh DG, Bigelow DB, Petty TL, Levine BE: Acute respiratory distress in adults. Lancet 2:319-323, 1967.

Ayuste EC, Chen H, Koustova E, Rhee P, Ahuja N, Chen Z, Valeri CR, Spaniolas K, Mehrani T, Alam HB: Hepatic and pulmonary apoptosis after hemorrhagic shock in swine can be reduced through modifications of conventional Ringer's solution. J Trauma 60:52-63, 2006.

Boura C, Caron A, Longrois D, Mertes PM, Labrude P, Menu P: Volume expansion with modified hemoglobin solution, colloids, or crystalloid after hemorrhagic shock in rabbits: effects in skeletal muscle oxygen pressure and use versus arterial blood velocity and resistance. Shock 19:176-182, 2003

Chudnofsky CR, Dronen SC, Syverud SA, Zink BJ, Hedges JR: Intravenous fluid therapy in the prehospital management of hemorrhagic shock: improved outcome with hypertonic saline $/ 6 \%$ dextran 70 in a swine model. Am J Emerg Med 7:357-363, 1989.

Cotton BA, Guy JS, Morris JA Jr, Abumrad NN: The cellular, metabolic, and systemic consequences of aggressive fluid resuscitation strategies. Shock 26:115-121, 2006.

Deb S, Martin B, Sun L, Ruff P, Burris D, Rich N, DeBreux S, Austin B, Rhee P: Resuscitation with lactated Ringer's solution in rats with hemorrhagic shock induces immediate apoptosis. J Trauma 46:582-588, 1999.

Deb S, Sun L, Martin B, Talens E, Burris D, Kaufmann C, Rich N, Rhee P: Lactated Ringer's solution and hetastarch but not plasma resuscitation after rat hemorrhagic shock is associated with immediate lung apoptosis by the up regulation of the box protein. J Trauma 49:47-53, 2000.

Demling RH: The pathogenesis of respiratory failure after trauma and sepsis. Surg Clin North Am 60:1373-1390, 1980.

Dillon J, Lynch LJ Jr, Myers R, Butcher HR Jr: The treatment of hemorrhagic shock. Surg Gynecol Obstet 122:967-978, 1966

Dong F, Hall CH, Golech SA, Philbin NB, Rice JP, Gurney J, Arnaud FG, Hammett M, Ma X, Flournoy WS, et al.: Immune effects of resuscitation with HBOC-201, a hemoglobin-based oxygen carrier, in swine with moderately severe hemorrhagic shock from controlled hemorrhage. Shock 25:50-55, 2006 .

Hashim R, Frankel H, Tandon M, Rabinovici R: Fluid resuscitation-induced cardiac tamponade. J Trauma 53:1183-1184, 2002.

Healey MA, Davis RE, Liu FC, Loomis WH, Hoyt DB: Lactated Ringer's is superior to normal saline in a model of massive hemorrhage and resuscitation. J Trauma 45:894-899, 1998 .

Jaskille A, Alam HB, Rhee P, Hanes W, Kirkpatrick JR, Koustova E: D-Lactate increases pulmonary apoptosis by restricting phosphorylation of bad and enos in a rat model of hemorrhagic shock. J Trauma 57:262-269, 2004.

Jaskille A, Koustova E, Rhee P, Britten-Webb J, Chen H, Valeri CR, Kirkpatrick JR, Alam HB: Hepatic apoptosis after hemorrhagic shock in rats can be reduced through modifications of conventional Ringer's solution. J Am Coll Surg 202:25-35, 2006

Knudson MM, Lee S, Erickson V, Morabito D, Derugin N, Manley GT: 
Tissue oxygen monitoring during hemorrhagic shock and resuscitation: a comparison of lactated Ringer's solution, hypertonic saline dextran, and HBOC-201. J Trauma 54:242-252, 2003.

Koustova E, Stanton K, Gushchin V, Alam HB, Stegalkina S, Rhee PM: Effects of lactated Ringer's solutions on human leukocytes. J Trauma $52: 872-878,2002$

Madigan MC, Kemp CD, Johnson JC, Cotton BA: Secondary abdominal compartment syndrome after severe extremity injury: are early, aggressive fluid resuscitation strategies to blame? J Trauma 64:280-285, 2008.

Masuno T, Moore EE, Cheng AM, Moore PK, Grant AR, Johnson JL: Prehospital hemoglobin-based oxygen carrier resuscitation attenuates post injury acute lung injury. Surgery 138:335-341, 2005.

Maxwell RA, Fabian TC, Croce MA, Davis KA: Secondary abdominal compartment syndrome: an underappreciated manifestation of severe hemorrhagic shock. J Trauma 47:995-999, 1999.

McCloskey CA, Kameneva MV, Uryash A, Gallo DJ, Billiar TR: Tissue hypoxia activates junk in the liver during hemorrhagic shock. Shock 22:380386, 2004.

Moon PF, Hollyfield-Gilbert MA, Myers TL, Kramer GC: Effects of isotonic crystalloid resuscitation on fluid compartments in hemorrhaged rats. Shock 2:355-361, 1994.

Nunn JF, Freeman J: Problems of oxygenation and oxygen transport during hemorrhage. Anesthesia 19:206-216, 1964.

Rhee P, Burris D, Kaufmann C, Pikoulis M, Austin B, Ling G, Harviel D, Waxman K: Lactated Ringer's solution resuscitation causes neutrophil activation after hemorrhagic shock. J Trauma 44:313Y319, 1998.

Sato H, Tanaka T, Kasai K, Kita T, Tanaka N: Role of p38 mitogen-activated protein kinase on renal dysfunction after hemorrhagic shock in rats. Shock 24:488-494, 2005

Sato H, Tanaka T, Kasai K, Kita T, Tanaka N: Role of p38 mitogen-activated protein kinase on cardiac dysfunction after hemorrhagic shock in rats. Shock 28:291-299, 2007.

Sauaia A, Moore FA, Moore EE, Moser KS, Brennan R, Read RA, Pons PT: Epidemiology of trauma deaths: a reassessment. J Trauma 38:185-193, 1995.

Shackford SR, Mackersie RC, Holbrook TL, Davis JW, HollingsworthFridlund P, Hoyt DB, Wolf PL: The epidemiology of traumatic death. A population-based analysis. Arch Surg 128:571-575, 1993.

Shires GT, Canizaro PC: Fluid resuscitation in the severely injured. Surg Clin North Am 53:1341-1366, 1973.

Stanford GG, Patterson CR, Payne L, Fabian TC: Hypertonic saline resuscitation in a porcine model of severe hemorrhagic shock. Arch Surg 124: $733-736,1989$.

(NHLBI) The NHLBI halts study of concentrated saline for patients with shock due to lack of survival benefit. NIH News [serial on the Internet]. Cited March 26, 2009. Last accessed August 1, 2009. Available at: http:// www.nih.gov/news/health/mar2009/nhlbi-26.htm.

Traverso LW, Lee WP, Langford MJ: Fluid resuscitation after an otherwise fatal hemorrhage: I. Crystalloid solutions. J Trauma 26:168-175, 1986.

Velasco IT, Pontieri V, Rocha e Silva M Jr, Lopes OU: Hyperosmotic $\mathrm{NaCl}$ and severe hemorrhagic shock. Am J Physiol 239:H664-H673, 1980.

Velasco IT, Rocha e Silva M, Oliveira MA, Silva RI: Hypertonic and hyperoncotic resuscitation from severe hemorrhagic shock in dogs: a comparative study. Crit Care Med 17:261-264, 1989.

Xu DZ, Lu Q, Adams CA, Issekutz AC, Deitch EA: Trauma-hemorrhagic shock- induced up-regulation of endothelial cell adhesion molecules is blunted by mesenteric lymph duct ligation. Crit Care Med 32:760-765, 2004. 